\title{
ON A CLASS OF COMPLETELY SEMISIMPLE INVERSE SEMIGROUPS
}

\author{
MARIO PETRICH
}

1. Introduction and summary. The semigroups in the title are precisely those whose principal factors are Brandt semigroups (with at most one exception which is a group). If we accept the latter as the building blocks, and try to build such semigroups out of Brandt semigroups, we first encounter the problem of (ideal) extensions of a Brandt semigroup by another; this problem has been studied in [4]. More generally, extensions of a Brandt semigroup by an arbitrary semigroup with zero have been investigated in [10]. Among extensions in general, those determined by a partial homomorphism are usually easier to handle and are thus of particular interest. Using a condition on idempotents, such extensions are studied in [7] for regular semigroups. Using this idea, a construction is given in [5] of certain regular semigroups using completely 0 -simple semigroups as building blocks.

The aim of this paper is to demonstrate that for the semigroups in the title, the conditions on idempotents can be replaced in certain cases by conditions on ideals (the latter are, generally, weaker and so our discussion would fail for completely semisimple semigroups). In Theorem 1, a simple necessary and sufficient condition in terms of ideals is given in order that an extension of a primitive inverse semigroup be determined by a partial homomorphism. This is used in Theorem 2 to establish a condition on ideals of a completely semisimple inverse semigroup in order that its idempotents form a tree. There are several consequences of these results.

All undefined concepts and symbols can be found in [2].

2. Extensions of a primitive inverse semigroup. An inverse semigroup $S$ with $0, S \neq 0$, is a primitive inverse semigroup if all its nonzero idempotents are primitive. Such semigroups are orthogonal sums of Brandt semigroups and conversely; several other characterizations of these semigroups can be found in [6, Corollaire 5.17], orthogonal sum is called "0-direct union" in $[2, \S 6.5]$. A function $\phi$ of a partial groupoid $A$ into a partial groupoid $B$ is a partial homomorphism if for any $x, y \in A$, whenever $x y$ is defined in $A$, then $(x \phi)(y \phi)$ is defined in $B$ and $(x y) \phi=(x \phi)(y \phi)$. If $V$ is a semigroup having an ideal $S$, then $V$ is an extension of $S$ by the Rees quotient semigroup $V / S$, or more gen-

Received by the editors June 23, 1969. 
erally, by any semigroup $T$ isomorphic to $V / S$. An extension $V$ of $S$ by $T$ is determined by a partial homomorphism $\phi$ if $\phi$ is a partial homomorphism of $T \backslash 0$ into $S$ such that for any $a, b \in T \backslash 0, c \in S$,

$$
a b=(a \phi)(b \phi) \quad \text { if } a b \in S, \quad a c=(a \phi) c, \quad c a=c(a \phi) .
$$

This is equivalent to the existence of a homomorphism $\psi$ of $V$ onto $S$ leaving every element of $S$ fixed [7, Proposition 2]; $\psi$ is an $S$-endomorphism of $V$. An ideal $I$ of a semigroup $S$ is categorical if for any $a, b, c \in S, a b, b c \notin I$ implies $a b c \notin I$ (quasi completely prime in [5], [6], $[8])$.

THEOREM 1. An extension $V$ of a primitive inverse semigroup $S$ is determined by a partial homomorphism if and only if $V$ has a categorical ideal $I$ such that $I \cap S=0$.

Proof. Necessity. Let $\psi$ be an $S$-endomorphism of $V$ and let $I=0 \psi^{-1}$. Then $I$ is an ideal and $I \cap S=0$ since $\psi$ leaves the elements of $S$ fixed. Let $a, b, c \in V$ be such that $a b, b c \notin I$. Then $(a \psi)(b \psi)=(a b) \psi$ $\neq 0$ and $(b \psi)(c \psi)=(b c) \psi \neq 0$ with $a \psi, b \psi, c \psi \in S$. Since $S$ is an orthogonal sum of Brandt semigroups, it is clear that 0 is categorical in $S$, so $(a b c) \psi=(a \psi)(b \psi)(c \psi) \neq 0$ and thus $a b c \notin I$. Consequently $I$ is categorical.

Sufficiency. Let $B=V / I$. Since $I \cap S=0$, we may consider $S$ as an ideal of $B$. If $\psi$ is an $S$-endomorphism of $B$, and we define $\phi$ on $V$ to agree with $\psi$ on $V \backslash I$ and map $I$ onto 0 , then it follows easily that $\phi$ is an $S$-endomorphism of $V$. The problem is thus reduced to constructing an $S$-endomorphism of $B$. Note that 0 is categorical in $B$. We will freely use properties of $S$ stemming from the fact that it is an or thogonal sum of Brandt semigroups. Let $a \in B$ and suppose that $a S \neq 0$. Hence $a x \neq 0$ for some $x \in S$ and there is a unique idempotent $e$ of $S$ such that $e a x \neq 0$. But then $e a \in S \backslash 0$. Thus there is an unique idempotent $f$ of $S$ such that $e a f=e a$. It follows that $a f \in S \backslash 0$ which implies that $e$ is the unique idempotent of $S$ with the property $e a f=a f$. Consequently $e$ and $f$ are the unique idempotents of $S$ for which $e a=a f \neq 0$. For such an element a define $a \psi=e a=a f$, and let $x \psi=0$ if $x \in B$ and $x S=0$. Then $\psi$ is a single-valued function mapping $B$ onto $S$ and leaving the elements of $S$ fixed.

Let $a, b \in B$. Suppose first that $a b S \neq 0$. Then $a \psi=e a \neq 0, b \psi=b f \neq 0$ and $(a b) \psi=(a b) g \neq 0$ for some idempotents $e, f, g$ of $S$. It follows that $b g \neq 0$ which by uniqueness implies that $f=g$. Further, $e a \neq 0, a b \neq 0$, $b f \neq 0$ imply $e a b f \neq 0$ since 0 is categorical in $B$, so $e(a b f)=a b f$ and thus

$$
(a b) \psi=a b g=a b f=(e a)(b f)=(a \psi)(b \psi) .
$$


Suppose next that $a b S=0$. If $a S=0$ or $b S=0$, then $(a \psi)(b \psi)=0$ $=(a b) \psi$. If $a S \neq 0$ and $b S \neq 0$, then $a \psi=e a$ and $b \psi=b f$ as above, so that

$$
(a b) \psi=0=e(a b f)=(e a)(b f)=(a \psi)(b \psi)
$$

since $a b S=0$. Therefore $\psi$ is an $S$-endomorphism.

Recall that an inflation of a semigroup $S$ is an extension of $S$ by a semigroup $T$ with $T^{2}=0$ determined by a partial homomorphism $[2, \S 3.2$, Exercise 10]. The next corollary shows that condition (b) [8, Corollary 2 to Theorem 2] can be omitted.

Corollary 1. A semigroup $V$ is an inflation of a primitive inverse semigroup if and only if $V^{2}$ is a primitive inverse semigroup and 0 is categorical in $V$.

Proof. Necessity. Let $V$ be an inflation of a primitive inverse semigroup $S$ and $\psi$ be an $S$-endomorphism of $V$. Then $S=S^{2} \subseteq V^{2} \subseteq S$ so that $V^{2}=S$. Let $a, b, c \in V$ be such that $a b \neq 0, b c \neq 0$. Then $a b$, $b c \in S \backslash 0$ and thus $(a \psi)(b \psi)=(a b) \psi=a b \neq 0$ and similarly $(b \psi)(a \psi) \neq 0$. But then $a b c=(a b c) \psi=(a \psi)(b \psi)(a \psi) \neq 0$. Consequently 0 is categorical in $V$.

Sufficiency. In Theorem 1 take $I=0$.Then $V$ is an inflation of $V^{2}$.

CoROllary 2. Let $S$ be a primitive inverse semigroup and let $T$ be a semigroup having 0 as its categorical ideal and no other proper ideals. Then an extension $V$ of $S$ by $T$ is determined by a partial homomorphism if and only if 0 is categorical in $V$.

Proof. Necessity. The ideal $I$ in Theorem 1 is either 0 or $\left(T \backslash 0_{T}\right) \cup 0_{S}$ (the zeros of $T$ and $S$, respectively) since $I$ can be considered as an ideal of $V / S$. In the first case 0 is categorical in $V$, and in the second case $V$ is an orthogonal sum of $S$ and $T$, and the hypothesis implies that 0 is categorical in $V$.

Sufficiency. This follows from Theorem 1 without any restriction on $T$.

3. Completely semisimple inverse semigroups. A semigroup $S$ is completely semisimple if each principal factor of $S$ is completely (0-) simple; in particular, an inverse semigroup $S$ is completely semisimple if and only if each principal factor is a Brand t semigroup with at most one exception which is a group. A poset $T$ is a tree if for any $e, f, g \in T$, $e \geqq f, e \geqq g$ implies that either $f \geqq g$ or $g \geqq f$. For a semigroup $S$, let $E_{S}$ denote the poset of its idempotents, and for $a \in S$, let $J(a)$ be the principal ideal generated by $a, J_{a}=\{b \in S \mid J(a)=J(b)\}, I(a)=$ $J(a) \backslash J_{a}$. 
LEMMA 1. In a completely semisimple semigroup $S$, the condition: if $e, f, g \in E_{S}, e \geqq f, e \geqq g, J(f)=J(g)$, then $f=g$ is equivalent to:

(C) if $J(a) \subseteq J(b)$, then there exists a partial homomorphism $\phi: J_{b} \rightarrow J_{a}$ such that for any $x \in J_{a}, y \in J_{b}, x y \in J_{a}$ implies $x y=x(y \phi)$ and $y x \in J_{a}$ implies $y x=(y \phi) x$.

PROOF. This follows readily from [3, Théorèmes 2.12 and 2.17].

Hence a completely semisimple semigroup $S$ in which $E_{S}$ in a tree satisfies the two conditions in Lemma 1.

Lemma 2. Let $S$ be a completely semisimple inverse semigroup satisfying condition (C) above. If $J(a) \subseteq J(b) \subseteq J(c)$ and $\phi: J_{c} \rightarrow J_{b}$, $\psi: J_{b} \rightarrow J_{a}, \tau: J_{c} \rightarrow J_{a}$ are partial homomorphisms in condition (C), then $\phi \psi=\tau$, and for every idempotent $e \in J_{c}$, we have $e \geqq e \phi$.

Proof. First let $e \in J_{c}$ and $f \in J_{b}$ be arbitrary idempotents. Then $J(f) \subseteq J(e)$ so $f=u e v$ for some $u, v \in S$. Letting $z=f u$ and $g=z^{-1} z$, we get $f=z g e v$, so that $g e \in J_{b}$. Since $J(b) / I(b)$ is a Brandt semigroup or a group and $g(g e)=g e$ is an idempotent, we must have $g=g e$. Using the hypothesis, we obtain $g=g e=g(e \phi)$ which implies that $g=e \phi$. Thus $e \geqq e \phi$; analogously $e \phi \geqq e \phi \psi$ and $e \geqq e \tau$. Hence $e \geqq e \phi \psi$ and $e \geqq e \tau$ where $e \phi \psi, e \tau \in J_{a}$, which by Lemma 1 implies that $e \phi \psi=e \tau$. Now for any $x \in J_{c}$, we obtain

$$
\begin{aligned}
x \phi & =\left(x x^{-1} x\right) \phi=\left[\left(x x^{-1}\right) \phi\right](x \phi)=\left(x x^{-1}\right)\left[\left(x x^{-1}\right) \phi\right](x \phi) \\
& =x x^{-1}(x \phi)=x\left(x^{-1} x\right) \phi .
\end{aligned}
$$

Using the same kind of formula on $x\left(x^{-1} x\right) \phi$ as an element of $J_{b}$, we get

$$
x \phi \psi=x\left(x^{-1} x\right) \phi\left[(x \phi)^{-1}(x \phi)\right] \psi=x\left(x^{-1} x\right) \phi\left(x^{-1} x\right) \phi \psi=x\left(x^{-1} x\right) \phi \psi ;
$$

analogously $x \tau=x\left(x^{-1} x\right) \tau$. But $\left(x^{-1} x\right) \phi \psi=\left(x^{-1} x\right) \tau$ which implies that $x \phi \psi=x \tau$ and thus $\phi \psi=\tau$.

THEOREM 2. In a completely semisimple inverse semigroup $S$, all ideals of $S$ are categorical if and only if $E_{S}$ is a tree.

Proof. Necessity. Let $e, f, g \in E_{S}$ have property $e>f, e>g$. If $J(f) \neq J(f g) \neq J(g)$, then $f e, e g \notin J(f g)$ and the hypothesis implies that $f g=f e g \notin J(f g)$, a contradiction. Hence we may suppose that $J(g) \subseteq J(f)$. Now $I(g)$ is either a categorical ideal of $S$ or is empty. In either case, $f e, e g \notin I(g)$ since $f e=f$ and $J(g) \subseteq J(f)$, and $g e=g \notin I(g)$. The hypothesis then implies that $f g=f e g \notin I(g)$ so $f g \in J_{o}$. Since $f g$ and $g$ are idempotents, $(f g) g=f g \in J_{\theta}$, and since $J(g) / I(g)$ is either a 
Brandt semigroup or a group, it follows that $f g=g$. Consequently $f \geqq g$ which proves that $E_{S}$ is a tree.

Sufficiency. Let $a, b, c \in S$ be arbitrary. Then $J(a b) \subseteq J(b)$ and $J(b c) \subseteq J(b)$, so by Lemma 2 , for any $e \in J_{b} \cap E_{S}$ there exist $f \in J_{a b} \cap E_{S}$ and $g \in J_{b c} \cap E_{S}$ such that $e \geqq f$ and $e \geqq g$. By the hypothesis either $f \geqq g$ or $g \geqq f$. We suppose that $f \geqq g$ so that $J(b c) \subseteq J(a b)$; the case $g \geqq f$ is treated similarly. Let $\phi: J_{b} \rightarrow J_{a b}, \psi: J_{a b} \rightarrow J_{b c}$ be the partial homomorphisms in condition (C), then by Lemma $2, \phi \psi: J_{b} \rightarrow J_{b c}$ is the required partial homomorphism. Hence

$$
\begin{aligned}
(a b) \psi & =\left(a b b^{-1} b\right) \psi=\left[\left(a b b^{-1}\right)(b \phi)\right] \psi=\left(a b b^{-1}\right) \psi(b \phi \psi), \\
b c & =b b^{-1} b c=(b \phi \psi)\left(b^{-1} b c\right) .
\end{aligned}
$$

Since $J(b c) / I(b c)$ is a Brandt semigroup or a group, we may write the elements of $J_{b c}$ as triples, say $(a b) \psi=(x ; i, j), b c=(y ; k, l)$. Then (2) and (3) imply that $b \phi \psi=(z ; k, j)$ for some $z$. Further, using (1) in Lemma 2, we get

$$
(a b) \psi=a b\left[(a b)^{-1}(a b)\right] \psi=a b\left[\left(b^{-1} \phi\right)\left(a^{-1} a b\right)\right] \psi=a b\left(b^{-1} \phi \psi\right)\left(a^{-1} a b\right) \psi
$$

which together with $(a b) \psi, b^{-1} \phi \psi \in J_{b c}$ implies that $a b\left(b^{-1} \phi \psi\right) \in J_{b c}$. Consequently

$$
\begin{aligned}
a b c & =a b\left[b^{-1}(b c)\right]=\left[a b\left(b^{-1} \phi \psi\right)\right](b c)=(a b) \psi\left(b^{-1} \phi \psi\right)(b c) \\
& =(x ; i, j)\left(z^{-1} ; j, k\right)(y ; k, l)=\left(x z^{-1} y ; i, l\right) \in J_{b c} .
\end{aligned}
$$

We have proved that $a b c \in J_{a b} \cup J_{b c}$ holds for any $a, b, c \in S$. Hence if $I$ is an ideal of $S$ and $a b, b c \notin I$, then $J_{a b} \cap I=J_{b c} \cap I=\square$ so that $J_{a b c} \cap I=\square$ and thus $a b c \notin I$. Therefore $I$ is categorical.

It is easy to see that if all principal ideals of an arbitrary semigroup $S$ are categorical, then also all ideals of $S$ are categorical. Hence in Theorem 2, "all ideals" can be substituted by "all principal ideals."

Corollary 1. A completely semisimple inverse semigroup all of whose ideals are categorical is a subdirect product of Brandt semigroups.

Proof. This follows from Theorem 2 and [3, Théorème 2.17].

A construction is given in [5], called "a tree of completely 0 -simple semigroups," which makes it possible to describe the structure of regular semigroups $S$ with 0 for which $E_{S}$ is a tree all of whose elements are of finite height (in the sense of [1]). For convenience call such a tree an " $L$-tree" and the resulting construction for inverse semigroups, an " $L$-tree of Brandt semigroups." The next corollary shows that for inverse semigroups, condition (c) in [5, Théorème 3.7] can be omitted. 
Corollary 2. A semigroup $S$ is an L-tree of Brandt semigroups if and only if $S$ is a completely semisimple inverse semigroup with zero all of whose principal ideals are categorical and of finite height in the poset of principal ideals of $S$.

In [9], a semigroup $S$ is called retractable if for every ideal $I$ of $S$, the semigroup $S$ is an extension of $I$ determined by a partial homomorphism. It follows from the theorem in [9] that the semigroups in Corollary 2 above are retractable. Indeed, retractable semigroups are described in [9] using the notion of yet another "tree," for convenience call it a $T$-tree. It is easy to see that: $L$-tree $\Rightarrow T$-tree $\Rightarrow$ tree, so that every "tree of completely 0 -simple semigroups" in the sense of $[5]$ is retractable.

\section{REFERENCES}

1. G. Birkhoff, Lattice theory, rev. ed., Amer. Math. Soc. Colloq. Publ., vol. 25, Amer. Math. Soc., Providence, R. I., 1948. MR 10, 673.

2. A. H. Clifford and G. B. Preston, The algebraic theory of semigroups, Vol. I, II, Math. Surveys, no. 7, Amer. Math. Soc., Providence, R. I., 1961; 1967, MR 24 \#A2627; MR 36 \#1558.

3. G. Lallement, Demi-groupes reguliers, Ann. Mat. Pura Appl. (4) 77 (1967), 47-130. MR 37 \#1505.

4. G. Lallement and M. Petrich, Extensions of a Brandt semigroup by another, (to appear).

5. - Structure d'une classe de demi-groupes réguliers, J. Math. Pures Appl. 48 (1969), 345-397.

6. - Décompositions I-matricielles d'un demi-groupe, J. Math. Pures Appl. (9) 45 (1966), 67-117. MR 33 \#5765.

7. M. Petrich, On extensions of semigroups determined by partial homomorphisms, Nederl. Akad. Wetensch. Proc. Ser. A. 69 Indag. Math. 28 (1966), 49-51. MR 33 $\# 5775$.

8. - Inflation of a completely 0-simple semigroup, Bull. Soc. Math. Belg. 19 (1967), 42-54. MR 36 \#1566.

9. E. J. Tully, Semigroups in which each ideal is a retract, J. Austral. Math. Soc. 9 (1969), 239-245.

10. R. J. Warne, Extensions of Brandt semigroups and applications, Illinois J. Math. 10 (1966), 652-660. MR $34 \# 2749$.

Pennsylvania State University, University Park, Pennsylvania 16802 